\title{
Vertical settling of pebbles in turbulent circumbinary discs and the in situ formation of circumbinary planets
}

\author{
Arnaud Pierens ${ }^{1}$, Richard P. Nelson ${ }^{2}$, Colin P. McNally ${ }^{2}$ \\ ${ }^{1}$ Université de Bordeaux, Observatoire Aquitain des Sciences de l'Univers, Allée Geoffroy St. Hilaire, 33165 Pessac, France \\ ${ }^{2}$ Astronomy Unit, Queen Mary University of London, Mile End Road, London, E1 4NS, UK
}

30 September 2021

\begin{abstract}
The inner-most regions of circumbinary discs are unstable to a parametric instability whose non-linear evolution is hydrodynamical turbulence. This results in significant particle stirring, impacting on planetary growth processes such as the streaming instability or pebble accretion. In this paper, we present the results of three-dimensional, inviscid global hydrodynamical simulations of circumbinary discs with embedded particles of $1 \mathrm{~cm}$ size. Hydrodynamical turbulence develops in the disc, and we examine the effect of the particle back-reaction on vertical dust. We find that higher solid-to-gas ratios lead to smaller gas vertical velocity fluctuations, and therefore to smaller dust scale heights. For a metallicity $Z=0.1$, the dust scale height near the edge of the tidally-truncated cavity is $\sim 80 \%$ of the gas scale height, such that growing a Ceres-mass object to a $10 M_{\oplus}$ core via pebble accretion would take longer than the disc lifetime. Collision velocities for small particles are also higher than the critical velocity for fragmentation, which precludes grain growth and the possibility of forming a massive planetesimal seed for pebble accretion. At larger distances from the binary, turbulence is weak enough to enable not only efficient pebble accretion but also grain growth to sizes required to trigger the streaming instability. In these regions, an in-situ formation scenario of circumbinary planets involving the streaming instability to form a massive planetesimal followed by pebble accretion onto this core is viable. In that case, planetary migration has to be invoked to explain the presence of circumbinary planets at their observed locations.
\end{abstract}

Key words: accretion, accretion discs - planet-disc interactions- planets and satellites: formation - hydrodynamics - methods: numerical

\section{INTRODUCTION}

Among the 11 circumbinary planets discovered by the Kepler mission, all but one orbit close to the dynamical instability limit, in a region where the structure of the circumbinary disc was expected to be highly perturbed during the planet formation stage. Circumbinary discs are therefore challenging environments for forming planets, such that they can provide a testbed that can constrain competing theories of planet formation. By extension, assuming that planets form similarly around binaries and single stars, testing models of planet formation around binaries can also provide hints about planet formation processes around single stars.

Two competing ideas have been proposed for forming circumbinary planets. The migration hypothesis assumes that circumbinary planets formed in the outer regions of the circumbinary disc, in a more accretion friendly environment, and then migrated inwards to the location where they are currently observed. Results of hydrodynamical simulations of the migration of planets in circumbinary discs (Nelson 2003; Pierens \& Nelson 2007, 2008a,b, 2013; Kley \& Haghighipour 2014, 2015; Penzlin et al 2021), which suggest migration stalling at the edge of the central cavity, are consistent with the observed locations of circumbinary planets. Moreover, the fact that mainly sub-Jovian circumbinary planets have been detected so far, and the only Jovian mass circumbinary planet that has been detected, Kepler-1647b (Kostov et al. 2016), has a long period ( 1100 days), is also in agreement with the expectations of hydrodynamical simulations (Pierens \& Nelson 2008), and hence with the migration scenario.

Although the migration hypothesis is an appealing scenario, it can not be excluded that circumbinary planets may be formed in-situ, either through planetesimal or pebble accretion. Regarding planetesimal accretion, previous studies have however shown that due to the tidal influence of the binary, planetesimals tend to acquire eccentricities large enough for the collisions to become erosive (Meschiari 2012 a,b; Paardekooper et al. 2012; Bromley \& Kenyon 2015).

The efficiency of pebble accretion as a means of forming circumbinary planets in situ has been examined by Pierens et al. (2020; hereafter Paper I). They showed that in circumbinary discs, the time needed for a Ceres-mass object to grow to the pebble isolation mass through pebble accretion is of the same order as the disc lifetime. Inefficient pebble accretion occurs because dust grains are puffed up and form a layer with finite thickness $H_{d} \gtrsim 0.1 H$, with $H$ being the gas scale height, resulting in a decrease of the pebble accretion efficiency by a factor of $H_{d} / r_{\text {acc }}$, where $r_{\text {acc }}$ is the accretion radius. This is a direct consequence of the turbulence operating in the disc, resulting from the parametric instability involving the resonant interaction between inertial-gravity waves and an eccentric mode in the disc (Papaloizou 2005; Barker \& Ogilvie 2014). Since circumbinary discs become eccentric through interaction with the central binary, they are good candidates for generating turbulence through the eccentric parametric instability. In paper I, we found that the resulting 
turbulence transports angular momentum outwards with an effective viscous stress parameter $\alpha \sim 5 \times 10^{-3}$, and the vertical velocity fluctuations are a few percent of the sound speed. In this initial study, we neglected the effects of the back-reaction from the grain particles onto the gas, and the primary aim of this paper to examine the effect of including the back-reaction. It is expected that turbulence will be weakened when dust feedback is included, as this decreases the sound speed of the dust-gas mixture and increases its inertia (Lin \& Youdin 2017). When turbulence originates from the Vertical Shear Instability (VSI: Nelson et al. 2013), Lin (2019) indeed found that the particle back-reaction favours dust settling against turbulence when the dust-to-gas ratio is larger than the nominal value of $Z=0.01$, because a vertical gradient in the dust-to-gas ratio induces a buoyancy force that tends to stabilize the VSI (Lin \& Youdin 2017). In this work we examine how varying $Z$ influences the turbulence in the inner regions of a circumbinary disc, and what the implications are for forming the observed circumbinary planets in situ.

This paper is organised as follows. In Sect. 2, we describe the hydrodynamical model and numerical setup. In Sect. 3, we present the results of our 3D simulations of the vertical settling of pebbles in turbulent circumbinary discs, and discuss the impact on the turbulence and on planet formation when the particle back-reaction onto the gas is included. Finally, we draw our conclusions in Sect. 4.

\section{THE HYDRODYNAMIC MODEL}

\subsection{Governing equations}

\subsubsection{Gas component}

We solve the hydrodynamical equations for the conservation of mass, momentum, and internal energy in spherical coordinates $(r, \theta, \phi)$ (radial, polar, azimuthal), with the origin of the frame located at the centre of mass of the binary. For the gas component, these equations read:

$$
\frac{\partial \rho}{\partial t}+\nabla \cdot(\rho \mathbf{v})=0
$$

$\rho\left(\frac{\partial \mathbf{v}}{\partial t}+\mathbf{v} \cdot \nabla \mathbf{v}\right)=-\nabla P-\rho \nabla \Phi-\rho_{d} \mathbf{f}_{\mathbf{d}}$

$\frac{\partial e}{\partial t}+\nabla \cdot(e \mathbf{v})=-(\gamma-1) e \nabla \cdot \mathbf{v}+Q_{c o o l}$,

where $\rho$ is the density, $P$ the pressure, $\mathbf{v}$ the velocity, $e$ the internal energy, $\gamma$ the adiabatic index, which is set to $\gamma=1.4$. In the previous equation, $\mathbf{f}_{\mathbf{d}}$ represents the mutual drag force between the gas and the solids, and $\Phi$ is the gravitational potential of the binary, whose parameters match those of Kepler-16 (Doyle et al. 2011; see Table 1 ). We note that $\Phi$ includes the contribution from the indirect term, However, we do not include the gravitational back-reaction from the disc onto the binary, so the binary orbit remains fixed.

Compared to our previous work, we make use of a more realistic cooling scheme that assumes that the cooling rate $Q_{\text {cool }}$ in Eq. 3 is given by:

$Q_{\text {cool }}=-\rho c_{v} \frac{T-T_{0}}{t_{\text {cool }}}$,

where $c_{v}$ is the specific heat capacity at constant volume, $T_{0}$ the background disc temperature and $t_{\text {cool }}$ the cooling timescale. $t_{c o o l}$ is calculated every time step by considering the timescale for radiative
Table 1. Binary parameters for the Kepler-16 system (from Doyle et al. 2011).

\begin{tabular}{cc}
\hline \hline Parameter label & Kepler-16 \\
\hline$M_{1}\left(M_{\odot}\right)$ & 0.69 \\
$M_{2}\left(M_{\odot}\right)$ & 0.2 \\
$q_{\text {bin }}=M_{2} / M_{1}$ & 0.29 \\
$a_{\text {bin }}(\mathrm{AU})$ & 0.22 \\
$e_{\text {bin }}$ & 0.16 \\
\hline
\end{tabular}

loss of energy from a gaussian sphere of scale height corresponding to the gas scale height $H$. It is given by:

$t_{\text {cool }}=\frac{\rho c_{v} H \tau_{e f f}}{3 \sigma T^{3}}$,

where $\sigma$ is the Stefan-Boltzmann constant, and where $\tau_{\text {ef } f}$ is the effective optical depth which is given by:

$\tau_{\text {eff }}=\frac{3}{8} \tau+\frac{\sqrt{3}}{4}+\frac{1}{4 \tau}$.

Following Bae et al. (2016), the optical depth $\tau$ is calculated as $1 / \tau=1 / \tau_{\text {upper }}+1 / \tau_{\text {lower }}$ with:

$\tau_{\text {upper }}=\int_{z}^{z_{\max }} \rho\left(z^{\prime}\right) \kappa\left(z^{\prime}\right) d z^{\prime}$

and

$\tau_{\text {lower }}=\int_{z_{\text {in }}}^{z} \rho\left(z^{\prime}\right) \kappa\left(z^{\prime}\right) d z^{\prime}$,

where the opacity $\kappa$ computed using the Rosseland mean opacity of Zhu et al. (2009). We show in the left panel of Fig. 1 the cooling time for our initial disc model which has mass equivalent to the MMSN (see Sect. 2.3). We see that in the disc midplane $t_{\text {cool }} \Omega \in[1,100]$ such that we expect from the results of Paper I that this region is prone to the eccentricity-induced parametric instability.

\subsubsection{Solid component}

In this work, dust is modelled as a second fluid whose governing equations are given by:

$\frac{\partial \rho_{d}}{\partial t}+\nabla \cdot\left(\rho_{d} \mathbf{v}_{\mathbf{d}}\right)=0$

$\rho_{d}\left(\frac{\partial \mathbf{v}_{\mathbf{d}}}{\partial t}+\mathbf{v}_{\mathbf{d}} \cdot \nabla \mathbf{v}_{\mathbf{d}}\right)=-\rho_{d} \nabla \Phi+\rho_{d} \mathbf{f}_{\mathbf{d}}$,

where $\rho_{d}$ is the density of solids and $\mathbf{v}_{\mathbf{d}}$ is the velocity.

In paper I, we considered particles that were characterized by a constant value of the Stokes number. In order to make physically meaningful predictions, here we fix the particle size to a value $a=1 \mathrm{~cm}$. For this particle size and the initial disc model, the corresponding Stokes number is shown in the right panel of Fig. 2. Particles experience gas drag according to the classical formulae for the Epstein and Stokes regimes. In the Epstein regime, namely for grains such that $\lambda>4 a / 9$ where $\lambda$ is the mean free path of the gas, the drag force is given by:

$\mathbf{f}_{\mathbf{d}}=\frac{1}{t_{S}}\left(\mathbf{v}-\mathbf{v}_{\mathbf{d}}\right)$

with the stopping time

$t_{s}=\frac{\rho_{p} a}{\rho c_{s}}$, 


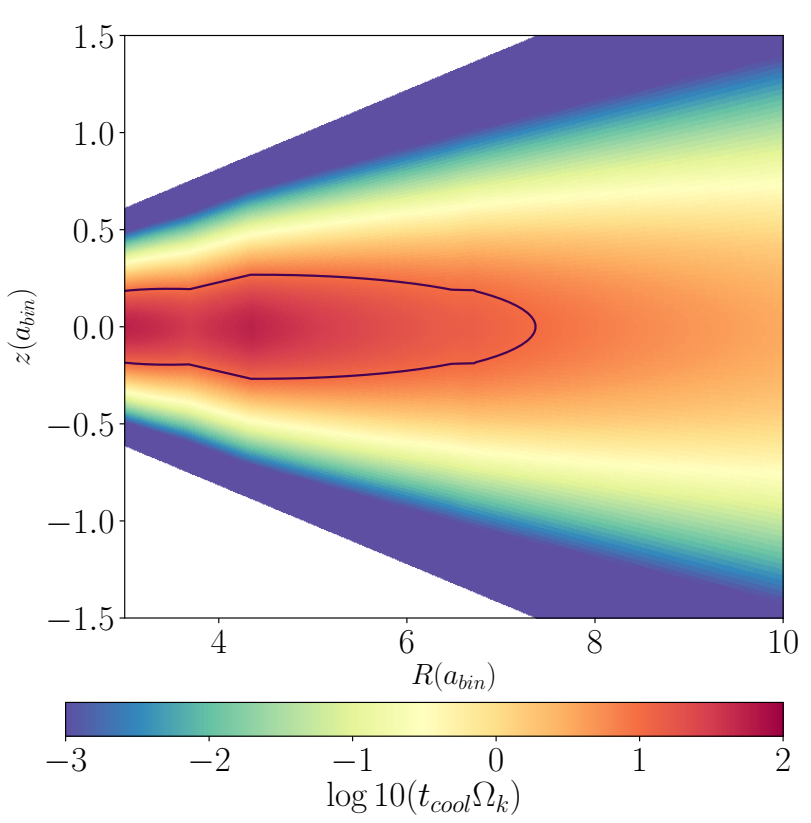

Figure 1. Two-dimensional R-Z distribution of the dimensionless cooling time for the initial disc model. The black line corresponds to the limit where $t_{\text {cool }} \Omega=1$.

where $c_{s}$ is the sound speed, $\rho_{p}$ is the material density, which is chosen to be $\rho_{p}=2 g . \mathrm{cm}^{-3}$. In the Stokes regime $(\lambda \leq 4 a / 9)$, the drag force is given by:

$\mathbf{f}_{\mathbf{d}}=\frac{1}{2} C_{D} \pi a^{2} \rho\left|\mathbf{v}-\mathbf{v}_{\mathbf{d}}\right|\left(\mathbf{v}-\mathbf{v}_{\mathbf{d}}\right)$,

where $C_{D}$ is the drag coefficient, which is given by:

$C_{D}= \begin{cases}24 \mathcal{R}_{e}^{-1} & \mathcal{R}_{e}<1 \\ 24 \mathcal{R}_{e}^{-0.6} & \mathcal{R}_{e}<1 \leq 800 \\ 0.44, & \end{cases}$

where $\mathcal{R}_{e}$ is the Reynolds number defined by:

$\mathcal{R}_{e}=\frac{2 a\left|\mathbf{v}_{\mathbf{d}}-\mathbf{v}\right|}{v_{p}}$,

where $v_{p}=\lambda c_{s} / 3$ is the molecular viscosity. The mean free path of the gas $\lambda=1 / n \sigma_{H_{2}}$, with $n=\rho /\left(\mu m_{H}\right)$ the number density of particles and $\sigma_{H_{2}}=10^{-15} \mathrm{~cm}^{2}$ the collisional cross-section of molecular hydrogen, is computed assuming a mean molecular weight of $\mu=2.4$.

\subsection{Numerical setup}

The simulations presented in this paper were performed using the multifluid version of FARGO3D (Benitez-Lamblay \& Masset 2016). Computational units are chosen such that the total mass of the binary is $M_{\star}=1$, the gravitational constant $G=1$, and the radius $R=1$ in the computational domain corresponds to the binary semi-major axis for the Kepler-16 system $\left(a_{\text {bin }}=0.22\right.$ au, see Table 1$)$. When presenting the simulation results, unless otherwise stated we use the binary orbital period $T_{\text {bin }}=2 \pi \sqrt{a_{\text {bin }}^{3} / G M_{\star}}$ as the unit of time.

The computational domain in the radial direction extends from $R_{\text {in }}=1.5 a_{\text {bin }}$ to $R_{\text {out }}=11 a_{\text {bin }}$ and we employ 716 logarithmically

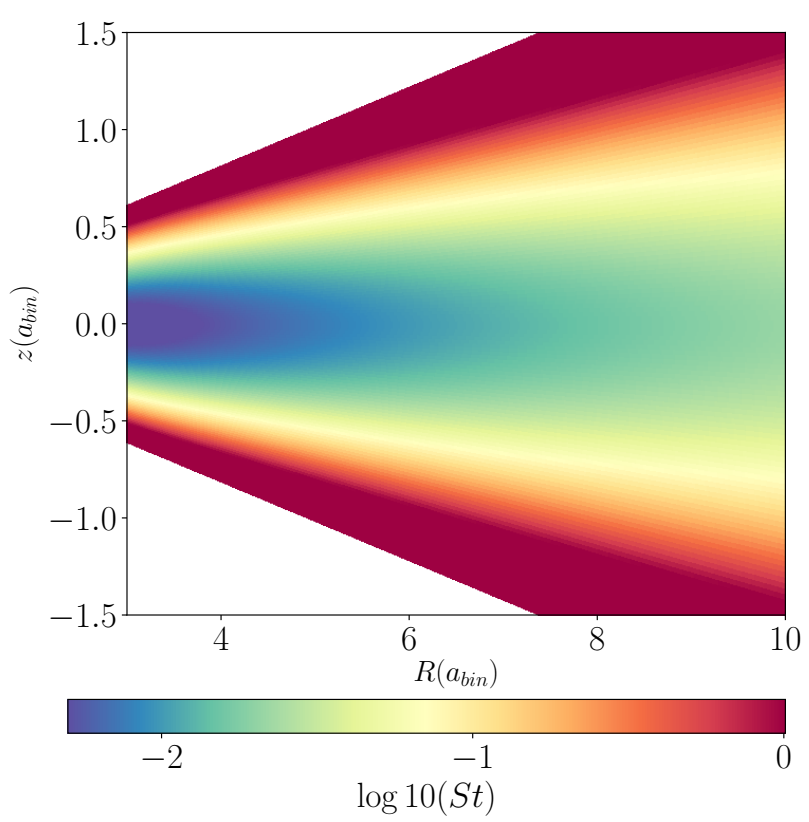

Figure 2. Two-dimensional initial R-Z distribution of the Stokes number of $\mathrm{cm}$-sized particles for the initial disc model.

spaced grid cells. In the azimuthal direction the simulation domain extends from 0 to $2 \pi$ with 700 uniformly spaced grid cells. In the meridional direction, the simulation domain covers 3.5 disc pressure scale heights above and below the disc midplane, and we adopt 144 uniformly spaced grid cells. Unstable inertial modes that are involved in the parametric instability have vertical wavelength $\lambda_{z} \sim$ $H$, and radial wavelength $\lambda_{R} \sim 0.6 H$ (Papaloizou 2005) and these are resolved by $\sim 18$ grid cells in the vertical direction and $\sim 11$ grid cells in the radial one. The numerical resolution we adopt in this work is therefore similar to that employed in Paper I.

\subsection{Initial conditions}

The initial radial profile of the sound speed, $c_{\mathrm{S}}$, is given by:

$c_{\mathrm{S}}(R)=h_{0}\left(\frac{R}{R_{0}}\right)^{q / 2}$,

where $R=r \sin \theta$ is the cylindrical radius and $h_{0}$ the disc aspect ratio at $R=R_{0}=1$. We adopt $h_{0}=0.05$ and $q=-1$ such that the aspect ratio, $h$, is constant with $h=h_{0}=0.05$.

The initial density and azimuthal velocity profiles of the gas are given by:

$\rho(R, z)=f_{\text {gap }} \rho_{0}\left(\frac{R}{R_{0}}\right)^{p} \exp \left(\frac{G M_{\star}}{c_{\mathrm{s}}^{2}}\left[\frac{1}{\sqrt{R^{2}+z^{2}}}-\frac{1}{R}\right]\right)$

and

$v_{\varphi}(R, z)=\left[(1+q) \frac{G M_{\star}}{R}+(p+q) c_{\mathrm{s}}^{2}-q \frac{G M_{\star}}{\sqrt{R^{2}+z^{2}}}\right]^{1 / 2}$,

where $z=r \cos \theta$ is the altitude and $\rho_{0}$ the density at $R=R_{0}$. The power-law index for the density is set to $p=-5 / 2$ so that the slope of the surface density profile $\Sigma$ corresponds to that of the MMSN, namely $\Sigma \propto R^{-3 / 2}$ (Hayashi 1981). In Eq. 17, $\rho_{0}$ is also defined 
such that it corresponds to the MMSN. $f_{\text {gap }}$ is a gap-function used to initiate the disc with an inner cavity (assumed to be created by the binary), and is given by:

$f_{\text {gap }}=\left(1+\exp \left[-\frac{R-R_{\text {gap }}}{0.1 R_{\text {gap }}}\right]\right)^{-1}$,

where $R_{\text {gap }}=2.5 a_{\text {bin }}$ is the analytically estimated gap size (Artymowicz \& Lubow 1994). The initial radial and meridional velocities are set to zero.

Regarding the dust component, the initial azimuthal velocity of the dust grains is Keplerian, and the initial radial and vertical velocities are set to be zero. The initial density of the grains is set to $\rho_{d}(R, z)=$ $\epsilon \rho(R, z)$, where $\epsilon$ is the dust-to-gas ratio. Initially, the dust-to-gas ratio therefore equals the metallicity $Z$ for which we consider initial values of $Z=0.01,0.1$.

\subsection{Boundary conditions}

For both the gas and solid components, we employ a closed radial boundary condition at both the inner and outer edges of the disc. At the outer boundary, we also make use of a wave-killing zone for $R>10$ to avoid wave reflection. Ordinarily we would adopt outflow conditions at the inner edge to allow mass to accrete onto the binary, and hence for a steady state of the gas structure to develop. In these 3D calculations we consider inviscid conditions, such that a steady structure in which viscous stresses and gravitational torques come into balance does not exist. Furthermore, the computational expense of running 3D simulations would not allow us to achieve such a steady state even if we relaxed the inviscid assumption. We therefore do not expect the inner boundary condition to play an important role in determining the outcome of our simulations.

At the meridional boundaries, an outflow boundary condition is used for the velocities, dust density, and gas internal energy, and for which all quantities in the ghost zones have the same values as in the first active zone, except the meridional velocity whose value is set to 0 if it is directed towards the disc midplane to prevent inflow of material. For the gas density, we follow Bae et al. (2016a) and maintain vertical stratification by solving the following condition for hydrostatic equilibrium in the meridional direction:

$\frac{1}{\rho} \frac{\partial}{\partial \theta}\left(c_{\mathrm{s}}^{2} \rho\right)=\frac{v_{\phi}^{2}}{\tan \theta}$.

\subsection{Diagnostics}

To analyse the results of the simulations, we define at each altitude $z=r \cos \theta$ the mean value $\left\langle Q>_{z}\right.$ of a quantity $Q$ by space averaging $Q$ over the full azimuth $2 \pi$ and over a small radial range around centred on a reference radius $R_{0}$ :

$<Q>_{z}=\frac{\int_{0}^{2 \pi} \int_{r_{\text {in }}}^{r_{\text {out }}} Q r \sin \theta d r d \phi}{\int_{0}^{2 \pi} \int_{r_{\text {in }}}^{r_{\text {out }}} r \sin \theta d r d \phi}$

where $r_{\text {in }}$ and $r_{\text {out }}$ correspond to the integration boundaries. Similarly, we define at each radius $r$ a local value for $Q$ obtained by space averaging $Q$ over the full azimuth $2 \pi$ and meridional direction $\theta \in\left[\theta_{\text {min }}, \theta_{\text {max }}\right]:$

$<Q>_{r}=\frac{\int_{0}^{2 \pi} \int_{\theta_{\text {min }}}^{\theta_{\max }} Q \sin \theta d \theta d \phi}{\int_{0}^{2 \pi} \int_{r_{\text {in }}}^{r_{\text {out }}} \sin \theta d \theta d \phi}$

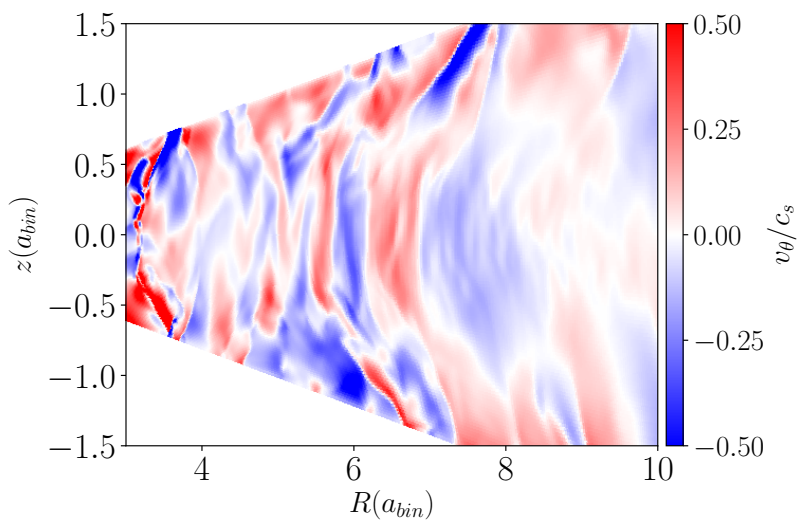

Figure 3. Contours of the gas meridional velocity $v_{\theta}$ in the $[R, Z]$ plane in the case where dust feedback is discarded
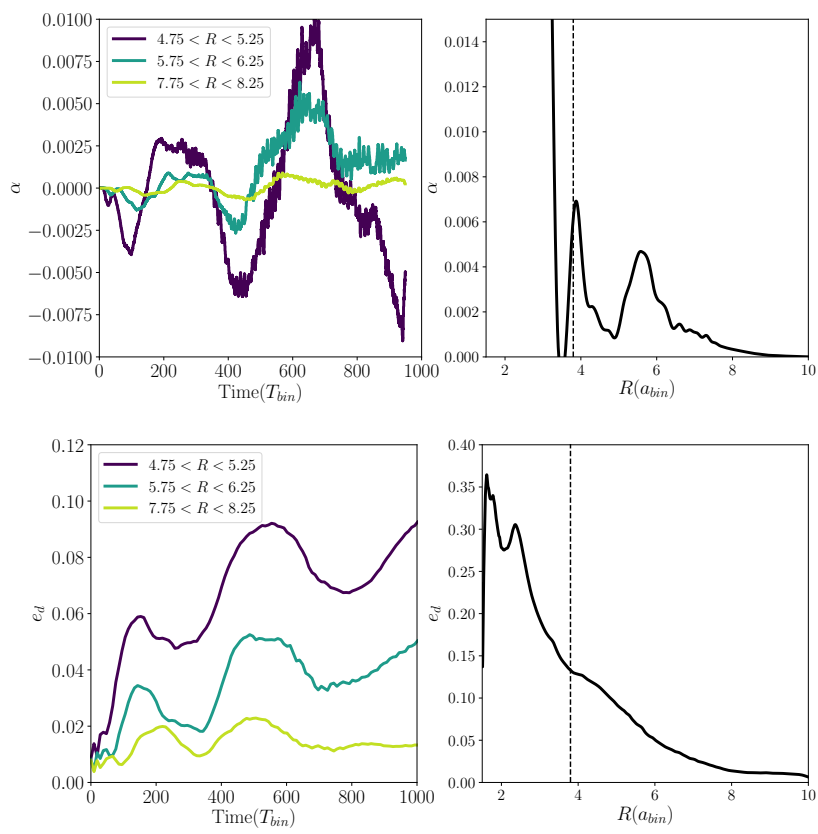

Figure 4. Left: $\alpha$ parameter (top) and disc eccentricity (bottom) as a function of time for the model without back-reaction included. Right: Radial profile of $\alpha$ (top) and $e_{d}$ (bottom) in the disc midplane. Here, a temporal average of 400 binary orbits has been used. The vertical dashed line corresponds to the location of the edge of the inner cavity.

We also denote by $\overline{\langle Q\rangle_{i}}$ the temporal average of the quantity $<Q>_{i}$ over time:

$\overline{<Q>_{i}}=\frac{1}{t_{2}-t_{1}} \int_{t_{1}}^{t_{2}}<Q>_{i} d t$

where $t_{1}$ and $t_{2}$ set the limits of integration in time. 


\section{RESULTS}

\subsection{Hydrodynamical turbulence driven by the disc eccentricity and the impact of the dust back-reaction}

Eccentric discs are unstable to a parametric instability involving the resonant interaction between inertial-gravity waves and the eccentric mode in the disc (Papaloizou 2005a; Barker \& Ogilvie 2014). The instability occurs whenever the inertial wave frequency $\omega$ matches the resonance condition:

$\omega \sim \frac{\Omega}{2}$

Both global (Papaloizou 2005b) and local (Wienkers \& Ogilvie 2018) simulations have found that the non-linear outcome of the instability is turbulence, with corresponding $\alpha$ viscous stress parameter that depends on the value for the disc eccentricity $e_{d}$. For instance, Papaloizou (2005) found $\alpha \sim 10^{-3}$ for $e_{d}=0.1$, whereas for smaller disc eccentricities $e_{d}=0.03, \alpha \sim 2 \times 10^{-4}$ (Wienkers \& Ogilvie 2018). In circumbinary discs where an eccentricity mode in the disc can grow as a result of the interaction with the central binary, our previous work demonstrated that this instability can also operate and ultimately lead to turbulence. For a central binary with parameters typical of Kepler-16 and constant cooling timescale $t_{\text {cool }}=\Omega^{-1}$, we confirmed the value for $\alpha$ to be correlated with the disc eccentricity, with $\alpha \sim 5 \times 10^{-3}$ at maximum.

For the more realistic thermal disc structure we consider here, turbulence is also found to set in, and with properties similar to what has been found in Paper I. In particular, the vertically elongated flow structures that were obtained in Paper I are reproduced, as revealed by looking at contours of the meridional velocity in Fig. 3. These vertical gas motions have typical radial wavelength of $\lambda_{R} \sim H$, consistent with what is expected from linear theory (Papaloizou 2005).

In the case where the effect of dust back-reaction is neglected, the joint temporal evolution of $\alpha$ and $e_{d}$ is shown, for different radial bins of the disc in the left panel of Fig. 4. To compute $\alpha$, we first average the Reynolds stress $T_{r \phi}=\rho \delta v_{r} \delta v_{\phi}$ over the azimuthal and meridional directions, where $\delta v_{r}$ and $\delta v_{\phi}$ are the radial and azimuthal velocity fluctuations. A local value for $\alpha$ is then given by $\alpha(r)=<T_{r \phi}>_{r} /<P>_{r}$ where $<P>_{r}$ is a mean pressure. Both $\alpha$ and $e_{d}$ undergo oscillations around a mean value because the binary longitude of pericentre maintains a constant value while the disc tends to precess with a finite libration amplitude. A clear trend for $\alpha$ to increase with disc eccentricity can be noticed, as expected for turbulence originating from the eccentricity induced parametric instability. This can also be observed in the right panel of Fig. 4 which depicts the azimutally averaged radial profiles of both quantities at the disc midplane, and further time-averaged over one precession period, which is estimated to $\sim 400 T_{\text {bin }}$. Near the cavity edge where particles are expected to concentrate, $\alpha \approx 0.01$ at maximum, which corresponds to a significant level of turbulence. At larger distances, however, where the disc eccentricity is much smaller than at the cavity edge, we see the turbulence drops off significantly such that particle stirring should also decrease there.

Although we will examine in detail the vertical distribution of particles later in the paper, we can anticipate that $\mathrm{cm}$-sized pebbles with $S t \approx 0.01$ located close to the cavity edge should experience significant stirring, such that solid particles would have more or less same scale height as the gas. The main consequence is that this would render pebble accretion inefficient, since the pebble accretion rate scales as $r_{H} / H_{d}$, where $r_{H}$ is the Hill radius. In particular, for a Ceres-mass body of mass $\sim 10^{-4} M_{\oplus}$, a simple estimate suggests
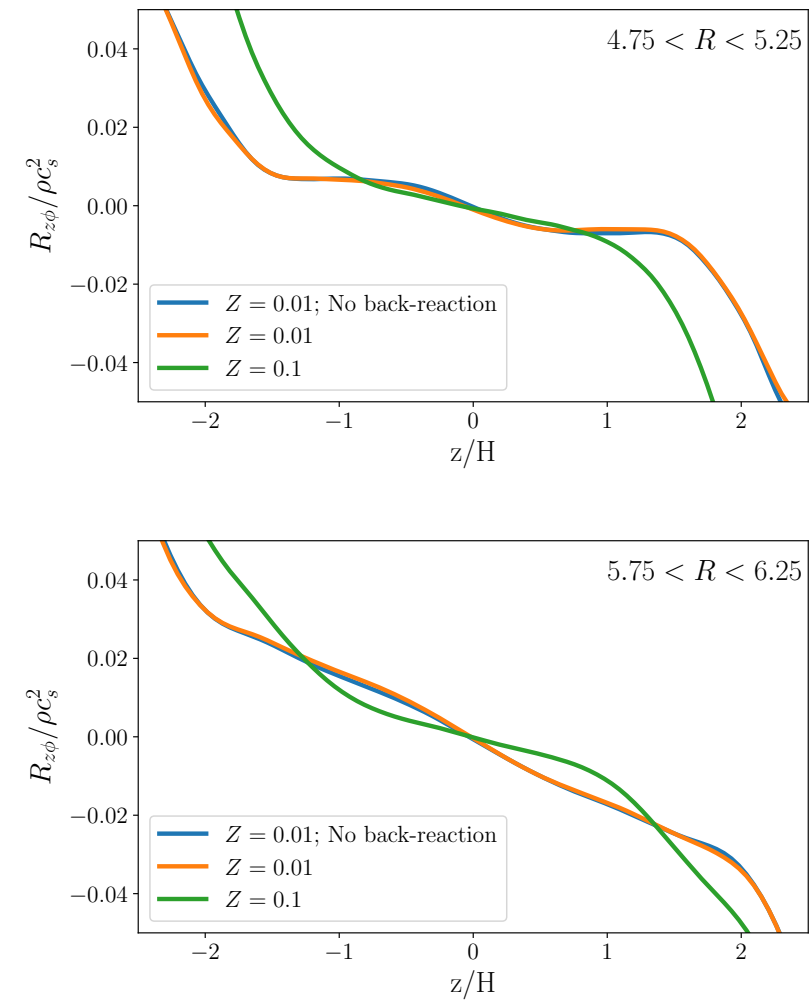

Figure 5. Top: Vertical profile of the vertical stress $R_{z \phi}$ in the region $R \in$ $[4.75,5.25]$ for the models with and without the effect of the dust backreaction onto the gas included. Bottom: same but for $R \in[5.75,6.25]$.

that the accretion rate would drop by a factor of $\sim 100$ compared to when the solid particles are more settled.

To assess the effect of the dust back-reaction on the eccentricityinduced parametric instability, we present in Fig 5 the mean vertical profile of the vertical stress:

$R_{z \phi}=\overline{\left\langle\rho \delta v_{\phi} \delta v_{z}>_{r}\right.}$

for our various models with/without back-reaction, where $\delta v_{z}$ is gas vertical velocity fluctuation. For anisotropic turbulence dominated by large scale vertical motions, as is the case here, the vertical stress is more relevant to the classical radial stress to quantity turbulence strength (Stoll \& Kley 2017; Lin 2019). Here, two different regions of the disc is considered for the radial average and a temporal average over 400 binary orbits is also used. It is clear that the primary effect of the particle back-reaction is to stabilize the instability, as a higher dust abundance results in a smaller vertical stress near the disc midplane. Compared to regions of the disc located at $R \approx 6$, the effect of the particle back-reaction and metallicity are slightly weaker at $R \approx 5$ because the dust scale height tends to be higher there (see Sect. 3.2). Hence, we can conjecture that a higher metallicity would tend to induce a smaller level of turbulence and therefore to favour dust settling, resulting in an enhanced efficiency of pebble accretion. As noted earlier, a similar result was found by Lin (2019) in the case of turbulence driven by the VSI. 
Table 2. Properties of the hydrodynamical turbulence as a function of parameters in the region $5.75<R<6.25$

\begin{tabular}{cccccccc}
\hline \hline Dust-to-gas ratio & Back-reaction & $H_{d} / H$ & $\begin{array}{c}<\overline{\delta v_{g, z}} \\
\left(c_{s}\right)\end{array}$ & $\alpha_{g, z}=<\overline{\delta v_{g, z}^{2}}>\tau_{c}$ & $\begin{array}{c}H_{d} / H \\
\text { (Eq. 26) }\end{array}$ & $\begin{array}{c}D_{d, z} / c_{s} H \\
\text { (Eq. 28) }\end{array}$ & $H_{d} / H$ \\
\hline$Z=0.01$ & No & 0.52 & 0.19 & 0.018 & 0.8 & 0.01 & 0.7 \\
$Z=0.01$ & Yes & 0.52 & 0.19 & 0.018 & 0.8 & 0.01 & 0.7 \\
$Z=0.1$ & Yes & 0.43 & 0.14 & 0.01 & 0.7 & 0.005 & 0.6 \\
\hline
\end{tabular}
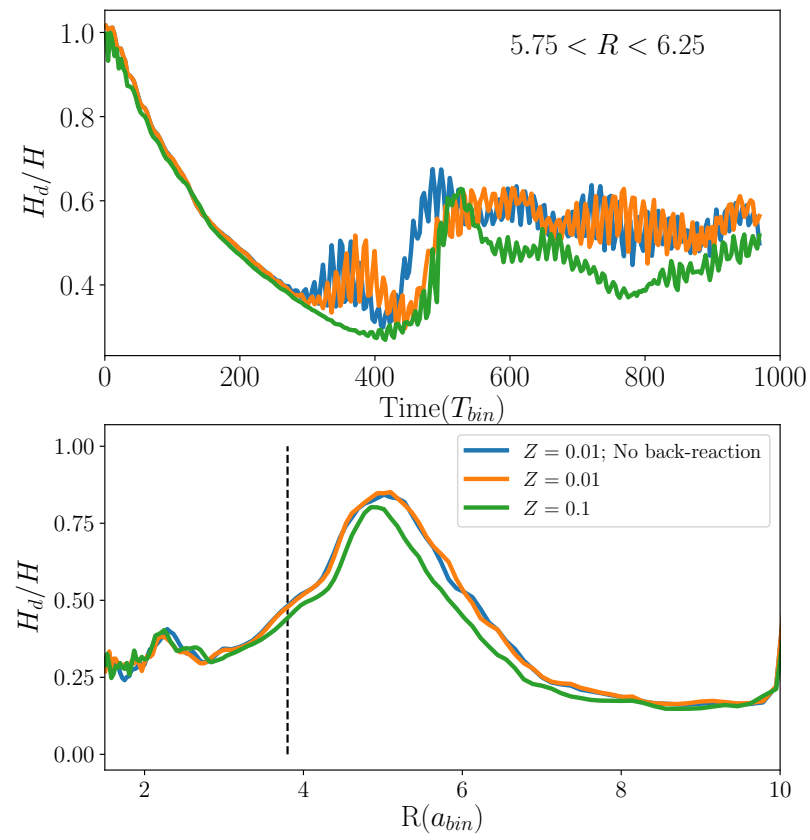

Figure 6. Top: Temporal evolution of the dust scale height relative to the the gas scale height. Bottom: Dust scale height as a function of radius. The vertical dashed line corresponds to the location of the edge of the inner cavity.

\subsection{Vertical distribution of cm-sized particles}

In order to examine the effect of the particle back-reaction in more detail, we now focus on the dynamical evolution of embedded $\mathrm{cm}-$ sized particles. We plot in the upper panel of Fig. 6 the temporal evolution of the dust scale height averaged between $R=5.75$ and $R=6.25$, relative to the gas scale height. The dust scale height $H_{d}$ is determined by fitting the dust distribution by a Gaussian function, which from Paper I appears to be a fairly good approximation. As expected, particles initially settle to the disc midplane until the onset of turbulence prevents further settling. From this point in time, the vertical distribution of particles reaches a steady state, as gravitational settling tends to balance turbulent diffusion. The vertical distribution of solids at the end of each run is shown in the top panel of Fig. 7, while the particle distribution at the disc midplane is presented in the bottom panel. As the particles that we consider here are marginally coupled to the gas, they become radially concentrated at the edge of the inner cavity and can be concentrated by the spiral waves launched by the binary.

Figure 6 shows that consistent with the above discussion about the effect of the solid abundance on the vertical stress, we obtain a smaller dust scale height for the run with higher metallicity $Z=0.1$. At equilibrium, we find that the difference in metallicity in the interval $R \in[5.75,6.25]$ is a factor of $\sim 12$ higher between the cases $Z=0.01$ and $Z=0.1$, which confirms that dust settling is favored in this latter case. However, the effect of the particle back-reaction appears to be negligible for a smaller metallicity $Z=0.01$. For each model, the values for $H_{d} / H$ at steady-state are listed in Table 2.

\subsection{Comparison with analytical estimates}

An issue that is worth examining is how the ratio $H_{d} / H$ obtained in our simulations at steady state compares with various analytical estimates that have appeared in the literature. An estimate obtained using diffusion theory (Dubrulle et al. 1995; Youdin \& Lithwick 2007) is given by:

$\frac{H_{d}}{H}=\sqrt{\frac{\alpha_{g, z}}{\alpha_{g, z}+S t}}$

where $\alpha_{g, z}=D_{g, z} / \Omega H^{2}$ is the dimensionless vertical diffusion coefficient of the gas. The gas vertical diffusion coefficient $D_{g, z}$ is given by:

$D_{g, z} \sim<\overline{\delta v_{z, g}^{2}}>\tau_{c o r}$,

where $\delta v_{z, g}$ is the vertical velocity dispersion of the gas and $\tau_{c o r}$ the correlation timescale of the hydrodynamical turbulence. $\tau_{c o r}$ can be determined by computing the time integral of the autocorrelation function (ACF) of the meridional velocity (Yang et al. 2009). This procedure has been used in Paper I and leads to $\tau_{\text {cor }} \sim 0.015 T_{\text {or }}$.

We plot the mean vertical velocity dispersion as a function of $z$ in the upper panel of Fig. 8. We immediately remark that this quantity: i) tends to increase (resp. decrease) when moving away from the disc midplane for high (resp. low) metallicities; ii) has a smaller value in the disc midplane for higher dust-to-gas ratios. For each model, the values for $\sqrt{\overline{\delta v_{g, z}^{2}}}$ at the disc midplane are reported in the fourth column of Table 2, while the resulting values for $\alpha_{g, z}$ are listed in the fifth column. Given that the $\mathrm{cm}$-sized particles located at $R \sim 6$ have $S t \sim 0.01$ (see Fig.2), plugging these latter values for $\alpha_{g, z}$ in Eq. 26 results in estimations for $H_{d} / H$ that are slightly overestimated (see Table 2). Moreover, using Eq. 26 would predict a minimum in the dust scale height profile at $R \approx 5$, as viscous stresses tend to be smaller there (see top right panel of Fig. 4).

Such a discrepancy may be attributed to the fact that Eq. 26 assumes that the dust diffusion coefficient in the vertical direction $D_{d, z}$ is equal to that of the gas, which is reasonable as long as the solids are strongly coupled to the gas. An alternative, more general estimation for $D_{d, z}$ can be obtained by assuming that at steady-state, the mass flux due to vertical settling balances the mass flux due to turbulent diffusion (Dubrulle et al. 1995; Zhu et al. 2015), which leads to (Riols \& Lesur 2018):

$D_{d, z}=-\frac{<\overline{\delta \rho_{d} \delta v_{z, d}}>}{\left(\bar{\rho}+\bar{\rho}_{d}\right)\left\langle\frac{\partial}{\partial z}\left(\frac{\bar{\rho}_{d}}{\bar{\rho}+\bar{\rho}_{d}}\right)\right\rangle}$.

The vertical profile of $D_{d, z}$ is shown in the bottom panel of Fig. 8, 
and the values for $D_{d, z}$ at the disc midplane are listed in Table 2. Overall, using the expression for $D_{d, z}$ given by Eq. 28 confirms the tendency for the dust diffusion coefficient to decrease as the dust-togas ratio is increased, although this leads to slightly smaller values in comparison to those given by Eq. 27. An alternative estimate for the dust scale height is then given by:

$$
\frac{H_{d}}{H}=\frac{1}{\sqrt{1+\frac{S t \Omega H^{2}}{D_{d, z}}}} .
$$

Employing the previous equation results in a better agreement with the dust scale height deduced from the simulations (see Table 2). For instance, for the model with $Z=0.1$ for which $H_{d} / H \approx 0.43$ at $R \approx 6$, using Eq. 26 gives $H_{d} / H \approx 0.7$ whereas Eq. 29 leads to $H_{d} / H \approx 0.6$. Moreover, looking at the vertical profile of $D_{d, z}$ for $R \in[4.75,5.25]$ shown in Fig. 9 , we see that in this region the dust diffusion is higher than further away in the disc, consistently with a higher dust scale height at this location. We note, however, that the estimates given by Eqs. 26 or 29 are not expected to be completely accurate, as they assume a uniform dust diffusion coefficient, whereas Fig. 8 shows this is not the case. Moreover, the diffusion model leading to these expressions assumes that the sizes of the turbulent eddies are smaller than $H$, while the hydrodynamical turbulence operating here is rather a mixture of small scale turbulence and coherent vertical motions occurring on scales larger than $H$.

\subsection{Planet growth times}

The smaller dust scale height obtained for higher dust-to-gas ratios confirms that the dust back-reaction tends to favour dust settling against the hydrodynamical turbulence. This is also illustrated by the bottom panel of Fig. 6 which shows the radial profile of $H_{d} / H$ time averaged over $400 T_{\text {bin }}$. At $R \approx 5$, namely close to the edge of the inner cavity, a value of $H_{d} / H \sim 0.8$ for $Z=0.1$ is obtained whereas $H_{d} / H \sim 0.85$ for $Z=0.01$. This implies that a large dust abundance is required to significantly decrease the particle scale height. At this location, a planet whose Hill radius is larger than the dust scale height has planet-to-star mass ratio $q_{p} \sim 1.7 \times 10^{-4}$ (or equivalently $\sim 50$ Earth masses) and would experience efficient pebble accretion. In other words, this means that a planet with $q_{p} \lesssim 1.7 \times 10^{-4}$ would accrete in the $3 \mathrm{D}$ regime for which the corresponding accretion rate, in the strong coupling limit, is given by (Lambrechts et al. 2019):

$$
\dot{M}_{p} \approx \frac{1}{4 \sqrt{2 \pi}} \frac{1}{\eta}\left(\frac{H_{d}}{H}\right)^{-1}\left(\frac{H}{r}\right)^{-1} F_{p e b} q_{p}
$$

where $F_{p e b}$ is the pebble flux for which we adopt a nominal value of $F_{p e b}=120 M_{\oplus} / M y r$, and

$\eta=-\frac{1}{2} h^{2}(p+q)$.

Following Pierens et al. (2020), we can compute the time, $t_{\text {growth }}$, required to grow a Ceres-mass planetesimal to a body with mass $m_{\text {target }}$ through pebble accretion. This is given by:

$t_{\text {growth }}=\int_{m_{\text {Ceres }}}^{m_{\text {target }}} \frac{d m}{\dot{M}_{p}}$.

Setting $m_{\text {target }}=10 M_{\oplus}$ results in $t_{\text {growth }} \sim 35 \mathrm{Myr}$, much longer than expected disc lifetimes. As mentionned earlier, this is because for planet masses $\lesssim 50$ Earth masses, pebble accretion proceeds in the 3D regime. At $R \approx 8$, however, $H_{d} / H \sim 0.25$ and the critical mass above which pebble accretion is not impacted by $3 \mathrm{D}$ effects becomes $q_{p} \sim 3 \times 10^{-6}$. Using Eqs. 30 and 32, we find that a core with $q_{p} \sim 3 \times 10^{-6}$ can be formed in $\sim 6 M y r$ in that case. Above this value for $q_{p}$, the accretion of pebbles is expected to be very efficient as it proceeds in $2 \mathrm{D}$, such that forming a $10 M_{\oplus}$ body within the disc lifetime seems very likely to be possible at large distances from the binary.

\subsection{Collision velocities}

Pebble accretion requires a pre-existing planetesimal seed which possibly formed through the streaming instability. The typical radius of planetesimals formed in models of streaming instability is $100 \mathrm{~km}$ (Schafer et al. 2017; Klahr \& Shreiber 2020). However, triggering the streaming instability at moderate solid-to-gas ratios requires the presence of large grains, which are not easily formed due to the bouncing and fragmentation barriers (Drazkowska \& Dullemond 2014). As turbulence is a source of impact velocities, a question of interest is whether the collisions speeds induced by the turbulence remain low enough to allow for sticking and grain growth.

In order to examine the locations in the disc where turbulence may eventually lead to collision speeds high enough to induce pebble fragmentation, we computed relative velocities between neighbouring particles as a function of distance from the central binary. For each grid cell located within a small patch of the disc $R_{i} \leq R \leq R_{O}$, $-0.01 \leq \pi / 2-\theta \leq 0.01$, we computed the relative velocities with respect to its neighbouring grid cells. The average of these relative velocities is then calculated for each grid cell and further time-averaged over 400 binary orbits. The spatial distribution of collisional velocities resulting from this procedure is shown in Fig. 10.

For each model, the relative velocity is $\sim 40 \mathrm{~m} \cdot \mathrm{s}^{-1}$ at the edge of the truncated cavity and decreases with increasing distance from the binary, reaching $\sim 11 \mathrm{~m} \cdot \mathrm{s}^{-1}$ at $R=9 \mathrm{a}_{\text {bin. }}$. These values can be compared to the estimate of the collisional velocity $v_{c}$ by Ormel $\&$ Cuzzi (2007) which predicts $v_{c} \approx \sqrt{3 \text { St } \alpha} c_{s}$ for small grains. From the right panel of Fig. 4, we have $\alpha \sim 0.01$ at maximum and $S t \sim 0.01$ at $R=5 a_{\text {bin }}$, whereas at $R=9 a_{\text {bin }}$ we have $\alpha \sim 0.001$ and $S t \sim 0.03$. Taken together, this leads to $v_{c} \sim 27 \mathrm{~m} \cdot \mathrm{s}^{-1}$ and $v_{c} \sim 9 \mathrm{~m} \cdot \mathrm{s}^{-1}$ at $R=5 a_{\text {bin }}$ and $R=9 a_{\text {bin }}$ respectively.

The critical velocity for dust fragmentation has been studied extensively both numerically and experimentally (Blum \& Wurn 2008; Zsom et al. 2010; Gundlach \& Blum 2015; Wada et al. 2013; Ueda et al. 2019), and is estimated to be between $1-10 \mathrm{~m} \cdot \mathrm{s}^{-1}$ for silicate particles and between $10-80 \mathrm{~m} \cdot \mathrm{s}^{-1}$ for icy aggregates. However, since we are mostly interested in regions inside the snow-line, which is approximately located at $2 \mathrm{AU}$ (Hayashi 1981), we can reasonably assume that the pebbles that we consider are silicate particles that were released consequently to the sublimation of icy pebbles. Fig. 10 reveals that turbulence may lead to the disruption of those $\mathrm{cm}$-sized silicate aggregates, particularly in the regions near the inner cavity. At these locations, it would be challenging to reach dust-to-gas ratios high enough to trigger the streaming instability. Also, the fact that collisions speeds are still higher than the fragmentation threshold velocity for a metallicity of $Z=0.1$ further suggests that the weakening of hydrodynamical turbulence due to a local increase in the concentration of dust would not be significant enough to allow for the growth of these grains.

Close to the inner cavity, the main implication of the fragmentation of cm-sized grains would be an increase in the population of smaller grains. However, such small grains may pass into the inner cavity and accrete onto the binary components along with the gas that flows into this region, as the outer edge of the cavity is not expected to trap small particles efficiently. This would tend to remove solids from the cavity edge, resulting in a less efficient planetesimal formation through the 

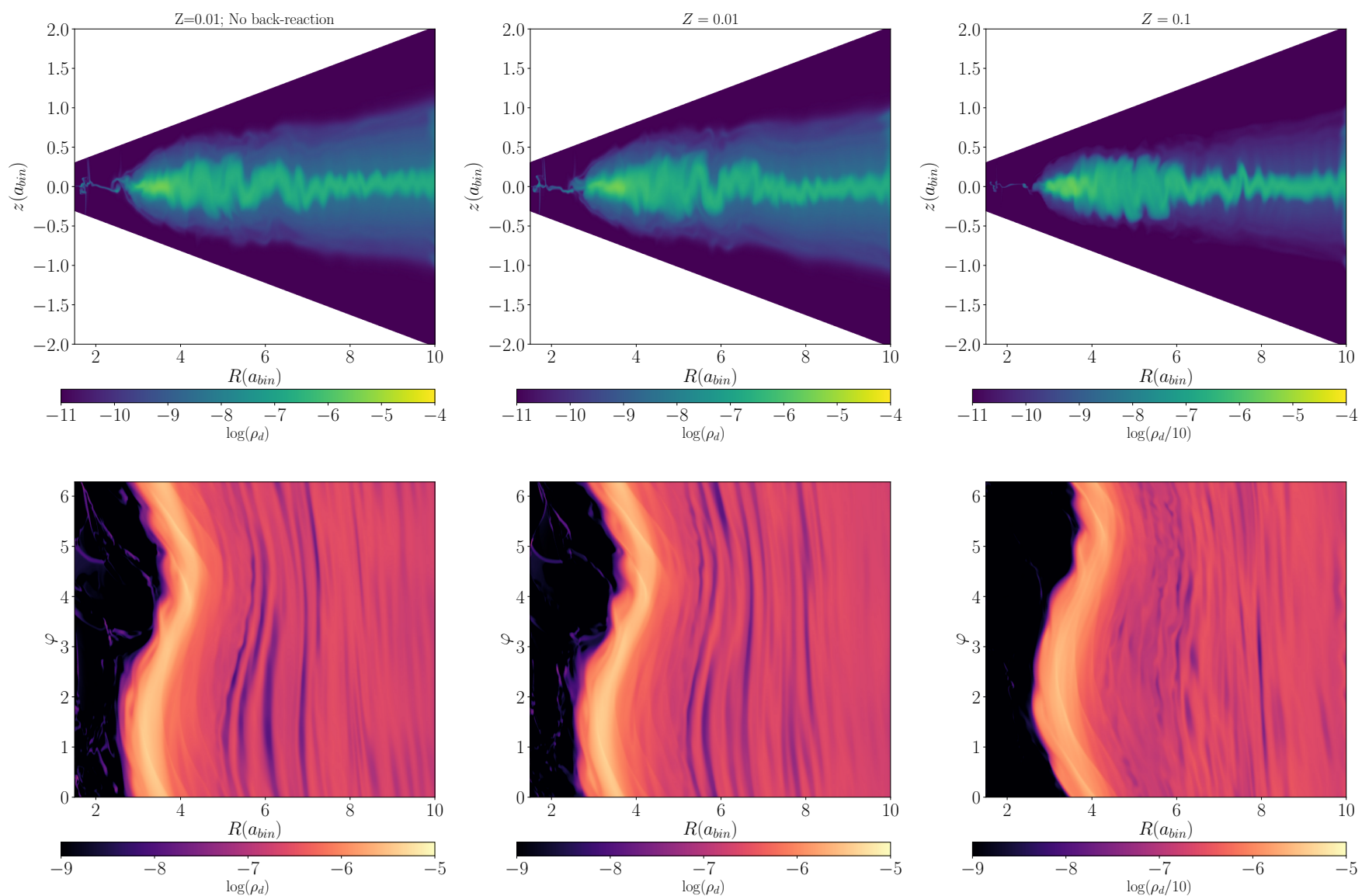

Figure 7. Top: vertical distribution of dust particles at $t=1000$ for the three models we considered. Bottom: density of solids in the disc midplane for the three models. In the case with $Z=0.1$, the dust density has been scaled by a factor of 0.1 for easier comparison.

streaming instability. The streaming instability indeed requires high solid abundances of $Z \sim 0.05$ to operate for particles with Stokes numbers of $S t \sim 10^{-3}$ (Yang et al. 2017). However, even if a massive planetesimal seed could be formed through the streaming instability involving these small grains, pebble accretion onto this core would be very inefficient in that case, due to the strong stirring by disc turbulence experienced by these small particles.

Further away from the binary, we remark that the collision velocities of $\mathrm{cm}$-sized grains are close to the fragmentation velocities derived from laboratory experiments. Considering distances even further than those that we include in the simulations, it is reasonable to suppose that grains could grow to sizes needed to trigger the streaming instability.

\subsection{Possible formation of dusty clumps}

Turning back to Fig. 7, we see from close inspection that compared to lower metallicities the case with $Z=0.1$ exhibits dusty clumps forming in the disc midplane in the region $R \in[4,6]$. There are basically two mechanisms that may be able to lead to the emergence of such clumps. Solids might accumulate at pressure maxima created by vortices emerging spontaneously from the hydrodynamical turbulence (Fromang \& Nelson 2005) or resulting from the Rossby Wave Instability (RWI; Lovelace et al. 1999; Li et al. 2000). Although not shown here, we examined the gas vorticity distribution and did not find any clear correlation between the vorticity and dust density dis- tributions. An alternative possibility is that these dust clumps result from the streaming instability (Youdin \& Goodman 2005; Youdin \& Johansen 2007) that tends to enhance the concentration of solids. Although our numerical resolution of $\approx 15$ grid cells per scale-height is probably insufficient to resolve the streaming instability whose most unstable wavelength is typically $\approx h H$, recent work (Chen \& Lin 2020; Urmurhan et al. 2020) has shown that in turbulent discs with $\alpha \approx 10^{-3}$, the most unstable wavelength might be shifted toward scales as large as $H$ (Johansen et al. 2007; Umurhan et al. 2020). In that case, our numerical resolution would be sufficient to witness the SI operating. The fact that dust clumps are not formed when the particle back-reaction is neglected is a further indication of the possibly important role of the mutual dust+gas drag on the formation of these clumps.

\section{CONCLUSIONS}

In this paper, we have presented the results of three-dimensional global hydrodynamical simulations of circumbinary discs subject to hydrodynamical turbulence driven by a parametric instability associated with the disc eccentricity (Papaloizou 2005; Barker \& Ogilvie 2014). Centimetre-sized solids modelled as a second fluid are introduced and we study the impact of turbulence on dust vertical settling. We also compare models with and without the effect of the 

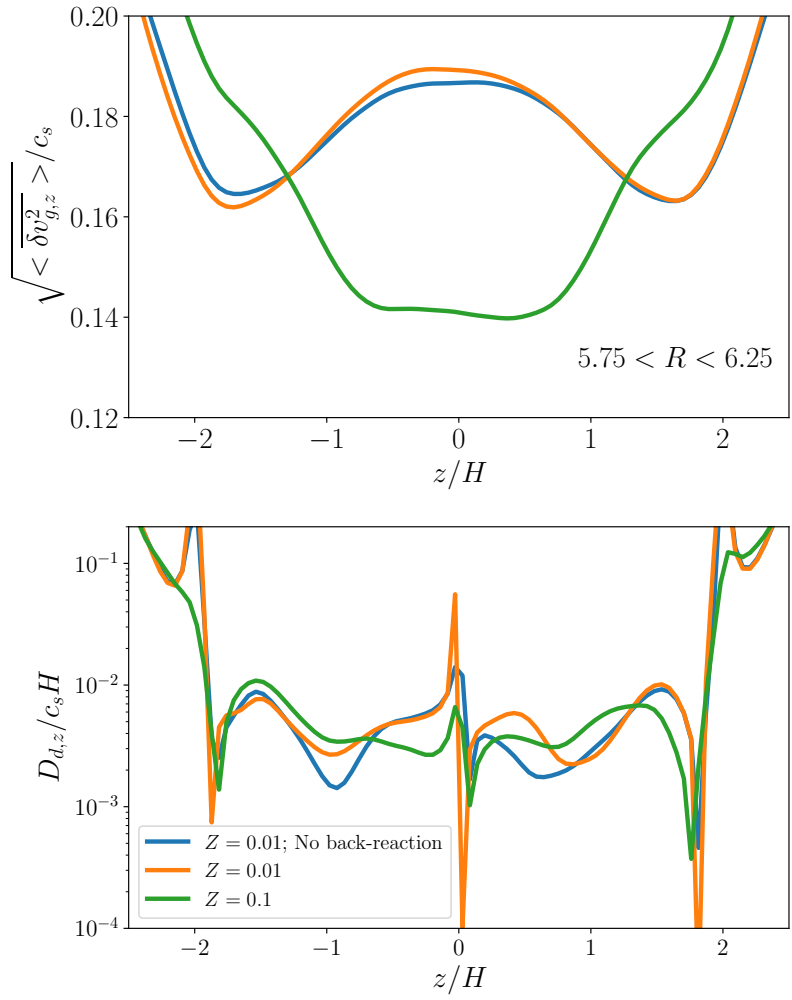

Figure 8. Top: mean gas vertical velocity fluctuations as a function of height for the three models we considered. Bottom: vertical profile of the dust diffusion coefficient computed using Eq. 28

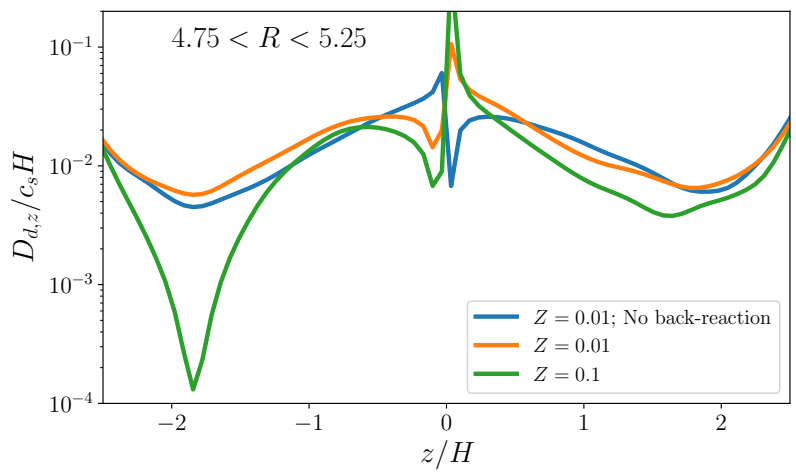

Figure 9. Vertical profile of the dust diffusion coefficient computed using Eq. 28 , for $R \in[4.75,5.25]$.

particle back-reaction onto the gas included, and examine the impact of varying the solid abundance.

In agreement with our previous findings (Pierens et al. 2020), the particle vertical profile reaches a quasi-stationary state once turbulent diffusion counterbalances gravitational settling. At steady-state, we find that a higher solid-to-gas ratio leads to a smaller dust scale height. This is a direct consequence of the effect of dust back-reaction onto the gas, which induces smaller vertical velocity fluctuations in the gas, resulting in a smaller dust vertical diffusion coefficient.

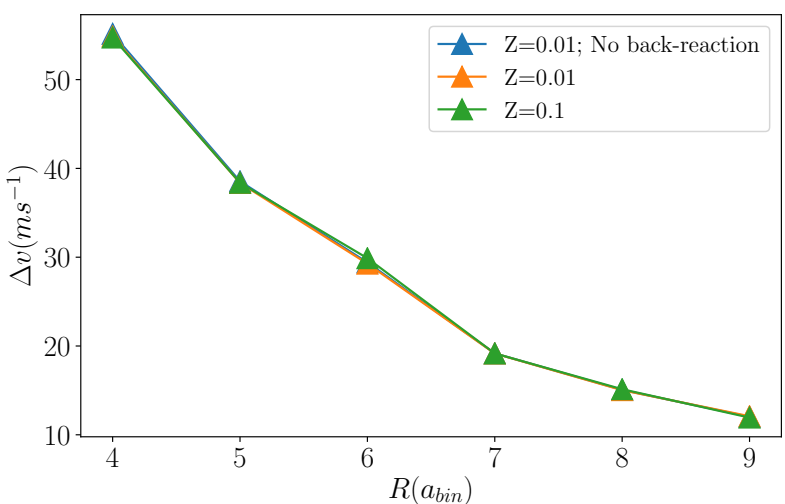

Figure 10. Relative velocities between grains as a function of distance from the central binary.

In the vicinity of the inner cavity formed by the binary, $\mathrm{cm}$-sized grains are significantly stirred by the turbulence, such that the dust and gas components have similar scale-heights. This renders pebble accretion inefficient in growing low-mass planetary cores, as the accretion rate is reduced by a factor of $r_{\text {acc }} / H_{d}$ compared to a laminar disc. Although increasing the dust-to-gas ratio slightly favours dust settling, we find that growing a Ceres-mass object to a $10 M_{\oplus}$ core in a disc with metallicity $Z=0.1$ still requires a time significantly longer than the disc lifetime. Growing such a planetary core, however, be possible at large distances from the binary, where the turbulence is weak enough so that pebble accretion onto a $1 M_{\oplus}$ planet becomes an essentially $2 \mathrm{D}$ process.

We also examined, in the different regions of the disc, how the impact velocities induced by the turbulence compare with the typical fragmentation velocities of silicate aggregates. Collision velocities are found to be significantly higher than the critical disruption velocity close to the inner binary, whereas further away from the binary these are comparable to the fragmentation velocity. This suggests the possibility of grain growth at large distances from the binary to sizes sufficient to trigger the streaming instability at moderate solid-to-gas ratios. Near the inner cavity, fragmentation would rather lead to an increase in the abundance of grains that have mm-sizes and smaller. However, it seems unlikely that such small grains can participate in the streaming instability because: i) these are expected to pass through the edge of the inner cavity, and ii) a high dust-to-gas ratio is required to trigger a streaming instability involving $\mathrm{mm}$-sized grains.

Taken together, these results suggest that forming a circumbinary planet, such as Kepler-16b, in-situ at the cavity edge, through a scenario that combines the streaming instability to build a seed planetesimal, followed by pebble accretion onto this seed object to form a planetary core that accretes gas, is very difficult to achieve. We simply note here that Kepler-16b orbits at a distance equivalent to $3.142 a_{\text {bin }}$ (Doyle et al. 2011), placing it right at the inner edge of the disc where the disturbance due to the binary and the hydrodynamical turbulence is at its strongest. This suggests that a more realistic scenario would involve formation of the planet further from the binary where the streaming instability and pebble accretion can operate more efficiently due to the lower levels of turbulence, followed by migration to the edge of the tidally truncated cavity formed by the binary. 


\section{ACKNOWLEDGMENTS}

Computer time for this study was provided by the computing facilities MCIA (Mésocentre de Calcul Intensif Aquitain) of the Universite de Bordeaux and by HPC resources of Cines under the allocation A0090406957 made by GENCI (Grand Equipement National de Calcul Intensif). CPM and RPN acknowledge support from STFC through grants ST/P000592/1 and ST/T000341/1.

\section{DATA AVAILABILITY}

The data underlying this article will be shared on reasonable request to the corresponding author.

\section{REFERENCES}

Artymowicz, P., \& Lubow, S. H. 1994, ApJ, 421, 651

Bae, J., Nelson, R. P., Hartmann, L., et al. 2016, ApJ, 829, 13

Barker, A. J., \& Ogilvie, G. I. 2014, MNRAS, 445, 2637

Benítez-Llambay, P., \& Masset, F. S. 2016, ApJS, 223, 11

Blum, J. \& Wurm, G. 2008, ARAA, 46, 21. doi:10.1146/annurev.astro.46.060407.145152

Chen, K. \& Lin, M.-K. 2020, ApJ, 891, 132. doi:10.3847/1538-4357/ab76ca

Doyle, L. R., Carter, J. A., Fabrycky, D. C., et al. 2011, Science, 333, 1602. doi:10.1126/science. 1210923

Drążkowska, J. \& Dullemond, C. P. 2014, A\& A, 572, A78. doi:10.1051/0004$6361 / 201424809$

Dubrulle, B., Morfill, G., \& Sterzik, M. 1995, Icarus, 114, 237

Hayashi, C. 1981, Progress of Theoretical Physics Supplement, 70, 35

Fromang, S. \& Nelson, R. P. 2005, MNRAS, 364, L81. doi:10.1111/j.17453933.2005.00109.x

Fromang, S. \& Papaloizou, J. 2006, A\& A, 452, 751. doi:10.1051/00046361:20054612

Gundlach, B. \& Blum, J. 2015, ApJ, 798, 34. doi:10.1088/0004$637 X / 798 / 1 / 34$

Johansen, A., Oishi, J. S., Mac Low, M.-M., et al. 2007, Nature, 448, 1022. doi: 10.1038 /nature 06086

Klahr, H. \& Schreiber, A. 2020, ApJ, 901, 54. doi:10.3847/1538-4357/abac58

Kley, W., \& Haghighipour, N. 2014, A\& A, 564, A72

Kley, W., \& Haghighipour, N. 2015, A\& A, 581, A20

Kostov, V. B., Orosz, J. A., Welsh, W. F., et al. 2016, ApJ, 827, 86

Li, H., Finn, J. M., Lovelace, R. V. E., \& Colgate, S. A. 2000, ApJ, 533, 1023

Lin, M.-K. 2019, MNRAS, 485, 5221. doi:10.1093/mnras/stz701

Lin, M.-K. \& Youdin, A. N. 2017, ApJ, 849, 129. doi:10.3847/15384357/aa92cd

Lovelace, R. V. E., Li, H., Colgate, S. A., \& Nelson, A. F. 1999, ApJ, 513, 805

Ormel, C. W. \& Cuzzi, J. N. 2007, A\& A, 466, 413. doi:10.1051/00046361:20066899

Meschiari, S. 2012, ApJ, 752, 71

Meschiari, S. 2012, ApJL, 761, L7

Mutter, M. M., Pierens, A., \& Nelson, R. P. 2017, MNRAS, 465, 4735

Nelson, R. P., Gressel, O., \& Umurhan, O. M. 2013, MNRAS, 435, 2610

Paardekooper, S.-J., Leinhardt, Z. M., Thébault, P., \& Baruteau, C. 2012, ApJL, 754, L16

Papaloizou, J. C. B. 2005a, A \& A, 432, 743

Papaloizou, J. C. B. 2005b, A\& A, 432, 757

Penzlin, A., Kley, W., Nelson, R. P. 2021, A\&A, 645, 68

Pierens, A., \& Nelson, R. P. 2007, A\& A, 472, 993

Pierens, A., \& Nelson, R. P. 2008, A\& A, 478, 939

Pierens, A., \& Nelson, R. P. 2008, A\&A, 483, 633

Pierens, A., \& Nelson, R. P. 2013, A\& A, 556, A134

Pierens, A., McNally, C. P., \& Nelson, R. P. 2020, MNRAS, 496, 2849. doi:10.1093/mnras/staa1550
Riols, A. \& Lesur, G. 2018, A\& A, 617, A117. doi:10.1051/0004$6361 / 201833212$

Schäfer, U., Yang, C.-C., \& Johansen, A. 2017, A\& A, 597, A69. doi:10.1051/0004-6361/201629561

Stoll, M. H. R., Kley, W., \& Picogna, G. 2017, A\&A, 599, L6. doi:10.1051/0004-6361/201630226

Ueda, T., Flock, M., \& Okuzumi, S. 2019, ApJ, 871, 10. doi:10.3847/15384357/aaf3a1

Umurhan, O. M., Estrada, P. R., \& Cuzzi, J. N. 2020, ApJ, 895, 4. doi:10.3847/1538-4357/ab899d

Wada, K., Tanaka, H., Okuzumi, S., et al. 2013, A\& A, 559, A62. doi:10.1051/0004-6361/201322259

Wienkers, A. F., \& Ogilvie, G. I. 2018, MNRAS, 477, 4838

Yang, C.-C., Mac Low, M.-M., \& Menou, K. 2009, ApJ, 707, 1233

Yang, C.-C., Johansen, A., \& Carrera, D. 2017, A\& A, 606, A80. doi:10.1051/0004-6361/201630106

Youdin, A. N. \& Goodman, J. 2005, ApJ, 620, 459. doi:10.1086/426895

Youdin, A. \& Johansen, A. 2007, ApJ, 662, 613. doi:10.1086/516729

Youdin, A. N., \& Lithwick, Y. 2007, Icarus, 192, 588

Zhu, Z., Hartmann, L., \& Gammie, C. 2009, ApJ, 694, 1045. doi:10.1088/0004-637X/694/2/1045

Zsom, A., Ormel, C. W., Güttler, C., et al. 2010, A\& A, 513, A57. doi:10.1051/0004-6361/200912976 